\title{
Discrimination of Cervical and Trochanteric Hip Fractures Using Radiography-Based Two-Dimensional Finite Element Models
}

\author{
Jérôme Thevenot ${ }^{*}$, , Pasi Pulkkinen ${ }^{1}$, Janne E. M. Koivumäki ${ }^{1}$, Volker Kuhn ${ }^{2,3}$, Felix Eckstein ${ }^{2,4}$ and \\ Timo Jämsä ${ }^{1,5}$ \\ ${ }^{I}$ Department of Medical Technology, University of Oulu, Finland \\ ${ }^{2}$ Institute of Anatomy, Ludwig-Maximilians-University, Munich, Germany \\ ${ }^{3}$ Department of Trauma Surgery and Sports Medicine, Medical University, Innsbruck, Austria \\ ${ }^{4}$ Institute of Anatomy and Musculoskeletal Research, Paracelsus Medical University, Salzburg, Austria \\ ${ }^{5}$ Department of Radiology, Oulu University Hospital, Finland
}

\begin{abstract}
Introduction: Predictors of fracture risk differ between cervical and trochanteric hip fractures. The aim of this experimental study was therefore to investigate whether two-dimensional (2D) finite element (FE) models, generated from standard radiographs, are able to predict and discriminate fracture types, originating from a simulated fall on the greater trochanter. Methods: A semi-automatic custom algorithm was applied to segment cortical and trabecular bone contours from radiographs of 49 female cadaver femora (mean age $80.7 \pm 10.3$ years). Two types of $2 \mathrm{D}$ FE models were generated, either one or four material properties assigned to the trabecular bone. The cartilage and soft tissue were also simulated, and the boundary conditions were mimicking the experiment. VonMises stress distributions within the trabecular bone were evaluated and the regions of maximum continuous stress patterns were determined. Results: The best fracture type prediction was obtained with the criterion that a cervical fracture was predicted if the maximum stress in trabecular bone was located at the superior part of the femoral neck and the maximum continuous stress pattern through the neck region; and in all other cases a trochanteric fracture was predicted. The two different models predicted $79.6 \%$ and $85.7 \%$ of the fracture cases correctly, in comparison with the actual failure type. Conclusion: Our results suggest that the cervical and trochanteric hip fractures can be discriminated with a satisfactory level of accuracy, using a relatively simple radiographybased 2D model. Based on the current experimental findings, the predictive power of these models should now be tested in clinical studies.
\end{abstract}

\section{INTRODUCTION}

Osteoporosis and related fractures in elderly people are an important public health issue worldwide, especially as their incidence increases with age, and the population of elderly people is growing [1]. Bone strength is related to mineral content, as assessed by bone densitometry, with the risk of fracture increasing proportionately with decrease in bone mineral density (BMD) [2]. Hip fracture is the most severe complication of osteoporosis and can lead to serious disability, a decrease in the quality of life, and high mortality [3]. A challenge of medical diagnostics is therefore to reliably identify people at risk before a fracture occurs.

Currently, the clinical diagnosis of osteoporosis is primarily based on the assessment of BMD at the proximal femur and lumbar spine using dual energy x-ray absorptiometry (DXA). However, DXA-based BMD has been shown to be insufficient to predict the individual risk of fracture $[4$,

*Address correspondence to this author at the Department of Medical Technology, University of Oulu, P.O. Box 5000, 90014 Oulu, Finland; Fax: +358-8-5376000; E-mail: jerome.thevenot@oulu.fi
5], most fractures occurring in people with a T-score $>-2.5$. This demonstrates an urgent need for more accurate tools for the assessment of osteoporotic fracture risk. It has been suggested that individual femora are at risk of a specific fracture type [6], and that the predictors of fracture risk differ between cervical and trochanteric fractures. According to previous studies, trochanteric fracture risk is primarily defined by BMD, while cervical fractures are more related to upper femur geometry, such as high neck-shaft angle (NSA) and long hip axis length [7-12].

Finite element (FE) modeling is a useful tool for studying the mechanical competence of the proximal femur and certain characteristics of hip fracture [13-21]. Most previous FE studies have relied on three-dimensional (3D) models, with data extracted from computed tomography (CT). Even if these models offer high accuracy and possibility to derive material properties at element level, they also have some disadvantages, as requiring an expensive method for capturing the 3D data, long computational times, and therefore, limited usefulness in clinical diagnostics. Also, these models have generally had a focus on fracture loads rather than the individual risk of a specific fracture type. 
It has been established that bone geometry has influence on the stress distribution in the proximal femur under given loading conditions [22]. Knowledge of the specific stress patterns leading to different hip fracture types may provide a better understanding behind the mechanism of hip fractures. More importantly, however, simple and universally available tools are required to determine the individual fracture risk with higher accuracy than DXA. If a geometric vulnerability to specific fracture types exists, the evaluation of fracture type specific thresholds using the FE method may be more reliable and efficient in predicting fracture risk than just a Tscore based on BMD alone. Lastly, fracture-type specific diagnostics may permit one to tailor prevention and therapy to the individual type of risk [23-25]. Previously, fracture risk has been predicted by DXA-based 2D models using the FE method [26, 27]. Radiography-based FE analysis might offer an improved low-cost alternative for the prediction of the individual risk of a specific fracture type and, consequently, for improved fracture risk assessment.

The aim of this experimental study on finite element model was therefore to investigate, whether the type of fracture (cervical or trochanteric) in a given femur, loaded in a configuration simulating a fall on the greater trochanter, can be accurately predicted using a relatively simple 2D model of the proximal femur, generated from a standard radiograph.

\section{MATERIALS AND METHODS}

\section{Study Sample and Mechanical Testing}

The cadaver femora were obtained from a larger experimental study $[8,28]$ from the Institute of Anatomy at the Ludwig Maximilians University of Munich (Germany). The subjects studied here were elderly women (mean age 80.7 , standard deviation 10.3 years) without bone diseases other than osteoporosis or osteopenia $[8,28]$. The femora were mechanically tested to failure, simulating a fall on the greater trochanter (Fig. 1). Mechanical testing procedures, preparation, and storage of the samples have previously been described in detail [28]. Briefly, the femoral shaft was positioned at $10^{\circ}$ from horizontal axis, and neck at $15^{\circ}$ internal rotation. Two halves of tennis ball were used to simulate cartilage contact in the femoral head. The load was applied to the greater trochanter through a pad simulating soft tissue at a constant loading speed of $6.6 \mathrm{~mm} / \mathrm{second}$, using a material testing machine (Zwick 1445, Ulm, Germany). The failure load was defined as the peak of the load-deformation curve. After the test, all 23 trochanteric fractures and a random sample of 26 cervical fractures were selected.

\section{Radiography and Image Segmentation}

Radiographs were taken using a Faxitron X-ray system (Model 43885A; Faxitron, Hewlett Packard, McMinnville, OR, USA) and the X-ray films were digitized together with a calibration scale using a scanner [8]. A set of geometrical parameters were measured from the radiographs to evaluate their ability to predict fracture type. Femoral neck axis lengths (FNALa and FNALb), neck-shaft angle (NSA), trochanteric width (TW), femoral head (HD), neck (ND) and shaft (FSD) diameters and femoral shaft cortex (FSC) and calcar femoral cortex widths (CFC) were measured as previously described [8, 9] using Image Tool software (version 3.00; University of Texas Health Science Center, San Antonio, TX, USA).

Semi-automatic custom algorithms were applied to extract the cortical vs. trabecular bone compartments based on grey levels using MATLAB (version 7.1, MathWorks, Natick, Massachusetts, USA). For each bone, two models were generated: (1) a model with only two Young's modulus

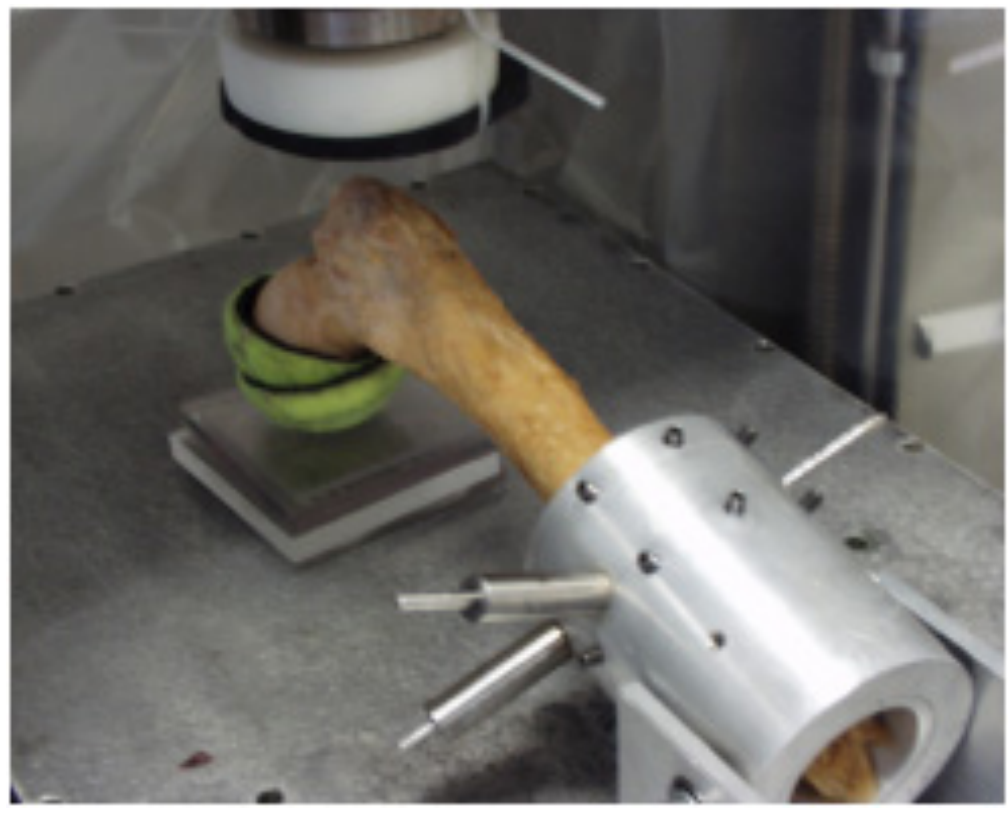

Fig. (1). Mechanical testing setup of the proximal femur that simulates a sideways fall on the greater trochanter. The femoral shaft was positioned at $10^{\circ}$ from horizontal axis, and neck at $15^{\circ}$ internal rotation. Two halves of tennis ball were used to simulate cartilage contact in the femoral head. The load was applied to the greater trochanter through a pad simulating soft tissue, using a constant loading speed of 6.6 $\mathrm{mm} / \mathrm{second}$. 
values (one for cortical bone and one for trabecular bone), and (2) a model with one Young's modulus value for cortical bone and four different values for different trabecular bone areas.

Contrast-limited adaptive histogram equalization (CLAHE) was applied for edge enhancement. Segmentation was done by semi-automatic selection of the anatomical contour of the cortical bone, using Hermite's splines for interpolation. The femoral head was modeled as a half-circle, and the thickness of the cortical bone was defined to be 1 $\mathrm{mm}$ at minimum. Finally, the femoral head, femoral neck, trochanteric, and subtrochanteric regions were separated for the generation of the model with four different Young's modulus values for trabecular bone. The four regions were separated using anatomical landmarks of the upper femur (Fig. 2). The femoral shaft was extended distally to obtain a standard size of the shaft length, defined as four times the femoral head diameter like in the experiment. The trochanter minor was used as an anatomic landmark for segmentation, but it was not included in the models for simplicity.

\section{Finite Element Models}

A two-dimensional reconstruction process was applied to obtain the 2D model using pre- and post-processing software FEMAP (version 9.2, E.S.P., Maryland Height, MO, USA). A femoral head surface with a thickness of $2 \mathrm{~mm}$ (Shepherd et al. 1999) was generated to simulate the cartilage. A loading pad of $9 \mathrm{~mm}$ thickness, simulating the soft tissue was also defined. Quad-elements with a mesh size of $2 \mathrm{~mm}$ were used; the models contained from 2000 to 2800 elements.

The material properties of the bone were assumed to be isotropic and linearly elastic. The Young's modulus of each various tissue region of the hip was taken from literature [2931]. The trabecular bone Young's modulus used for the femoral neck area was $780 \mathrm{MPa}, 900 \mathrm{MPa}$ for the femoral head area, $600 \mathrm{MPa}$ for the trochanteric region, and $300 \mathrm{MPa}$ for the subtrochanteric region [29-31], and a two-element transition area was used between the regions. The trabecular bone Young's modulus for the models with one material property was defined to be $780 \mathrm{MPa}$, as an average of the upper femur material properties. For the cortical bone, a Young's modulus of 14.2 GPa was used [32]. Poisson's ratio of 0.33 was selected for the bone tissues [33]. Young's modulus of $700 \mathrm{MPa}$ and $300 \mathrm{MPa}$ were used for the simulated soft tissue and cartilage during the mechanical testing setup, respectively.

Boundary conditions were chosen to simulate a fall on the greater trochanter during the mechanical test $[8,28]$. Loading was applied on the greater trochanter through the soft tissue with an angle of $10^{\circ}$ with the horizontal-axis (Fig. 1). A force of $1000 \mathrm{~N}$ was selected, based on the experimental studies, to indicate stress concentration under elastic deformation. The internal rotation of the bone used in experiment was ignored in this 2D study.

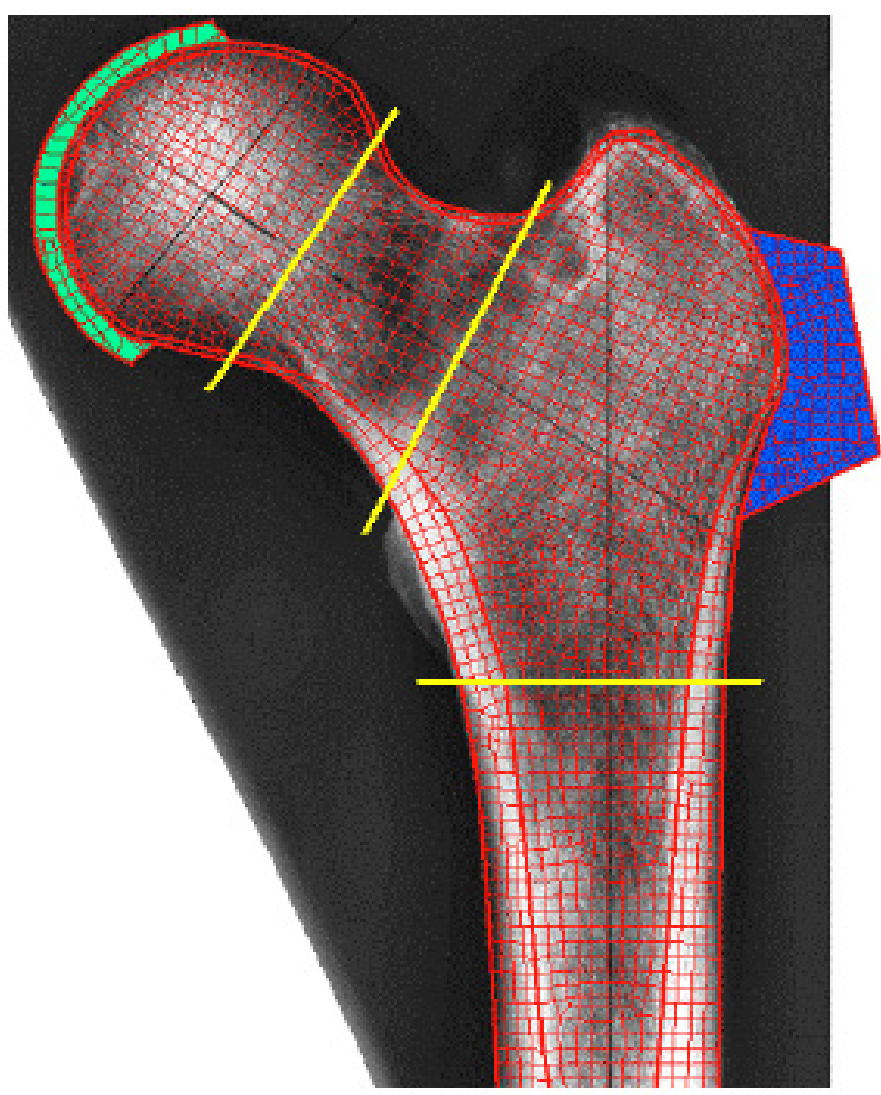

Fig. (2). Finite element mesh after segmentation and comparison with the original radiographic image. In green, the simulated cartilage and in purple the simulated soft tissue. The femoral head, neck, trochanteric and subtrochanteric/shaft regions are separated as shown with yellow lines. 
Table 1. Radiological Parameters of the Upper Femur Geometry and Experimentally Obtained Failure Load, Presented by Fracture Type. The Geometrical Parameters are Explained in Text

\begin{tabular}{|c|c|c|c|c|c|c|c|c|c|c|c|c|}
\hline Group & $\mathbf{N}$ & & $\begin{array}{c}\text { FSC } \\
(\mathrm{mm})\end{array}$ & $\begin{array}{c}\text { CFC } \\
(\mathbf{m m})\end{array}$ & $\begin{array}{l}\text { FSD } \\
(\mathbf{m m})\end{array}$ & $\begin{array}{l}\text { ND } \\
(\mathbf{m m})\end{array}$ & $\underset{(\mathbf{m m})}{\text { HD }}$ & $\begin{array}{c}\text { TW } \\
(\mathbf{m m})\end{array}$ & $\begin{array}{c}\text { FNALa } \\
(\mathbf{m m})\end{array}$ & $\begin{array}{c}\text { FNALb } \\
(\mathbf{m m})\end{array}$ & $\begin{array}{l}\text { NSA } \\
\text { (deg) }\end{array}$ & $\begin{array}{c}\text { Load } \\
(\mathrm{N})\end{array}$ \\
\hline \multirow{2}{*}{ Cervical } & \multirow{2}{*}{26} & Mean & 4.7 & 3.8 & 30.7 & 29.6 & 43.8 & 57.3 & 96.5 & 77.2 & 126.9 & 2830 \\
\hline & & SD & 1.6 & 1.2 & 1.9 & 1.9 & 2.5 & 3.1 & 5.9 & 5.5 & 5.3 & 802 \\
\hline \multirow{2}{*}{ Trochanteric } & \multirow{2}{*}{23} & Mean & 5.0 & 4.2 & 30.8 & 30.0 & 43.6 & 57.4 & 94.4 & $74.7\left(^{*}\right)$ & $121.4 * * *$ & $3526^{* *}$ \\
\hline & & SD & 1.2 & 1.1 & 2.6 & 1.9 & 1.6 & 3.0 & 4.0 & 4.6 & 6.0 & 937 \\
\hline
\end{tabular}

$\left(^{*}\right) \mathrm{P}<0.1, * * \mathrm{P}<0.01, * * * \mathrm{P}<0.001$, when compared with the cervical fractures.

$\mathrm{SD}$, standard deviation.

\section{Assessment of Fracture Type}

VonMises stress analysis [29] was performed for each model using NASTRAN (version NX; UGS, Plano, Texas, USA). VonMises stress distributions within the trabecular bone were evaluated and the regions of maximum continuous stress patterns were determined. Two different factors were defined: (1) location of the peak stress value, and (2) region of the maximum continuous stress pattern [22]. The best combination of these two factors to define the fracture type was determined.

\section{Repeatability of the Method}

The segmentation error was evaluated from five femora by superimposing the FE model to the original radiograph (Fig. 2). The maximum error was estimated to be less than $2 \mathrm{~mm}$. The repeatability was evaluated including the segmentation, the prediction of the hip fracture type, and the maximum level of VonMises stress, performing the full procedure 2 times for all 49 bones. Root-mean-square coefficient of variation of the maximum VonMises stress in the trabecular bone was calculated and was found to be $2.5 \%$. The fracture type prediction had a repeatability of $94 \%$ to $96 \%$ for the different models.

\section{Statistical Analysis}

Statistical analyses were performed using the SPSS statistical software (version 16.0, Chicago, IL., USA).

Independent $\mathrm{t}$-test was performed to study the differences in the geometrical variables and failure loads between the fracture types. Logistic regression analysis was used to find the best combination of geometrical parameters for the prediction of fracture type. $\mathrm{p}<0.05$ was considered statistically significant.

\section{RESULTS}

Geometrical parameters and failure loads by fracture types are shown in the Table 1. Cervical fracture cases had significantly higher NSA $(\mathrm{p}<0.001)$ and lower failure load $(\mathrm{p}<0.01)$ compared with the cases of trochanteric fracture.

The combination of NSA and FNALb yielded the best prediction of fracture type when all measured geometrical parameters were used as explanatory factors; $76.9 \%$ of cervical and $78.3 \%$ of trochanteric fractures were predicted correctly using the model with these two variables $(\mathrm{p}=$ 0.003) (Table 2).

The cervical fracture cases had typical VonMises stress distributions in finite element analysis, with the maximum stress in the superior neck area, and the maximum continuous stress pattern through the neck (Fig. 3A). The trochanteric cases displayed more variation (example in Fig. 3B). The best criterion for the prediction of hip fracture type was found to be as shown in Fig. (4):

- $\quad$ Checking first the location of the maximum VonMises stress level: if it is on the trochanteric area, the fracture is classified directly as trochanteric.

- If the maximum stress is located in the neck area, the VonMises stress pattern is also checked.

- The fracture is classified as cervical only if the maximum VonMises stress level is located on the superior femoral neck area and the maximum continuous stress pattern passes through the neck (Fig. 3A).

- $\quad$ All other cases are considered as trochanteric.

Table 2. Prediction of Fracture Type Using the Best Combination of Geometrical Parameters, and FE Model with One or Four Material Properties for Trabecular Bone. The Decision Criterion for the FE Models is Presented in Fig. (4)

\begin{tabular}{|c|c|c|c|c|c|c|}
\hline \multirow{3}{*}{ Experimental fracture } & \multicolumn{6}{|c|}{ Predicted Fracture Type } \\
\hline & \multicolumn{2}{|c|}{ Geometric model $^{1}$} & \multicolumn{2}{|c|}{ FE model $1^{2}$} & \multicolumn{2}{|c|}{ FE model $2^{3}$} \\
\hline & Cervical & Trochanteric & Cervical & Trochanteric & Cervical & Trochanteric \\
\hline Cervical & $20(76.9 \%)$ & $6(23.1 \%)$ & $22(84.6 \%)$ & $4(15.4 \%)$ & $23(88.5 \%)$ & $3(11.5 \%)$ \\
\hline Trochanteric & $5(21.7 \%)$ & $18(78.3 \%)$ & $6(26.1 \%)$ & $17(73.9 \%)$ & $4(17.4 \%)$ & $19(82.6 \%)$ \\
\hline
\end{tabular}

${ }^{1}$ Neck-shaft angle (NSA) and femoral neck axis length (FNALb)

${ }^{2}$ One homogeneous material property for trabecular bone

${ }^{3}$ Four different material properties for trabecular bone 

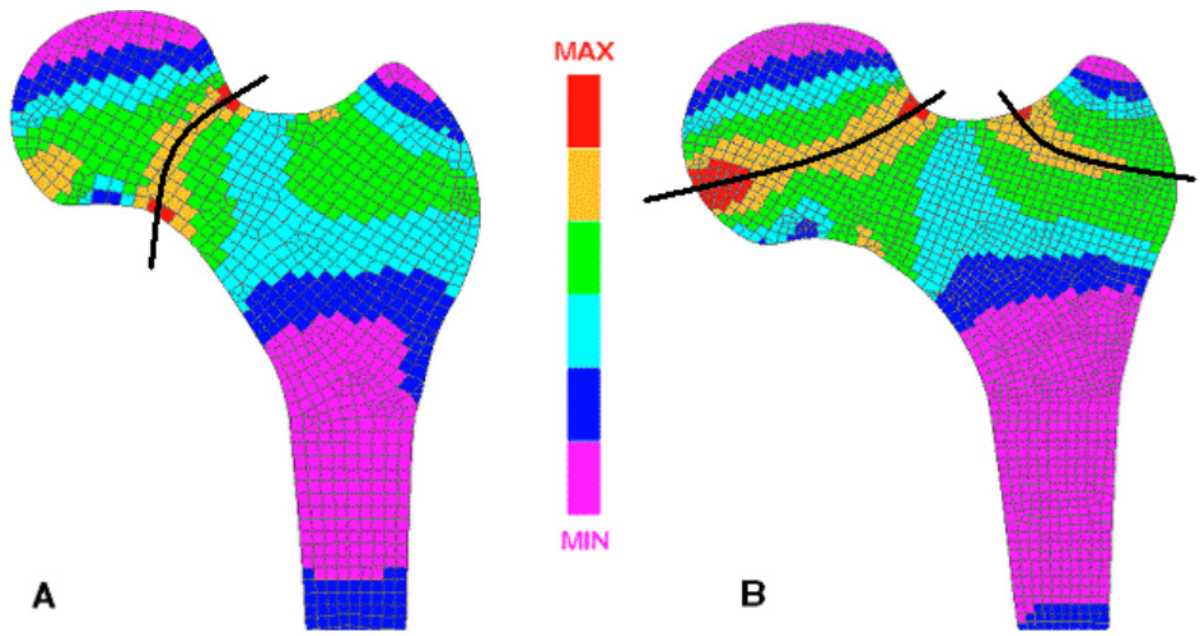

Fig. (3). VonMises stress distributions in the trabecular bone in cervical and trochanteric fracture cases. Finite element models with four Young's modulus values for trabecular bone. The black curves highlight the continuous stress concentration zones. MIN/MAX refer to minimum and maximum VonMises stress values for each bone. A. Cervical fracture. Maximum stress is located on the superior femoral neck and the maximum continuous stress pattern (uninterrupted pattern of red/orange elements) goes through the neck. B. Trochanteric fracture. Maximum stress is located on the neck area, and there is also stress concentration on the superior trochanteric area. However, the maximum continuous stress does not go through the femoral neck.

This procedure predicted the fracture type correctly in $79.6 \%$ of the cases $(84.6 \%$ cervical, $73.9 \%$ trochanteric fractures) using the models with homogeneous trabecular bone properties. $85.7 \%$ of all cases $(88.5 \%$ cervical, $82.6 \%$ trochanteric fractures) were accurately predicted using the models with heterogeneous material properties for the trabecular bone (Table 2).
In some bones, the prediction of the hip fracture type was not so straight forward, due to ambiguous stress patterns. One bone with a wide NSA was estimated to encounter a neck fracture, but showed a trochanteric fracture during the biomechanical experiment. Two femora, in contrast, had a small NSA and a trochanteric fracture was predicted, but experimentally the femora failed at the neck.

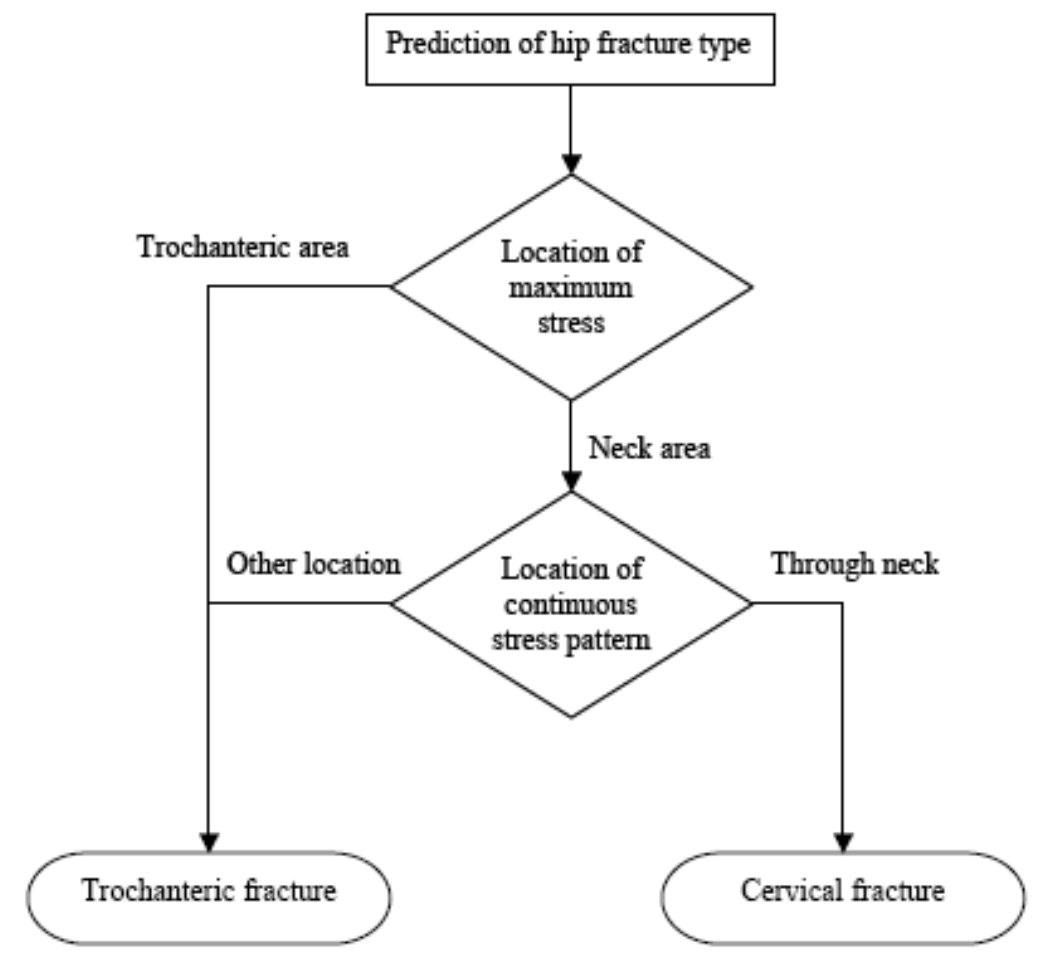

Fig. (4). Decision-making tree for cervical and trochanteric hip fractures based on the VonMises stress analysis in the trabecular bone. First, if the maximum stress is located in trochanteric area, the fracture is directly classified as trochanteric; if not, the location of the continuous stress pattern is checked. If the maximum continuous pattern passes through the neck area, the bone is classified as cervical fracture, and otherwise as trochanteric case. 


\section{DISCUSSION}

This study investigates the ability of a radiograph-based 2D FE model to discriminate hip fracture type (cervical or trochanteric) in the simulation of a fall on the greater trochanter. The results show that the cervical and trochanteric hip fractures can be discriminated with satisfactory accuracy using the VonMises stress distribution in the trabecular bone, even when a simple 2D model is used. This suggests that the method offers an efficient, less time-consuming tool for fracture type prediction applicable at lower cost and with a smaller radiation dose than required for CT.

The findings of this study are in agreement with previous studies that have demonstrated the importance of bone geometry in hip fracture type distribution, cervical fractures being more related to femoral geometry and trochanteric fracture risk being more strongly defined by $\operatorname{BMD}[9,12$, 28]. Here, the FE models showed somewhat better accuracy than the combination of the geometrical measures, suggesting that $\mathrm{FE}$ analysis yields a more comprehensive prediction by capturing the varying stress distribution caused by the shape of bone, whereas the measurement of a limited set of local geometrical parameters provides a rougher estimate for the shape.

Since homogeneous material properties were applied here, these models mainly reflect the impact of the upper femur geometry. Our findings suggest that even a simple 2D representation of the proximal femur can give some prognostic information concerning the assessment of the risk of a certain fracture type. The most important geometrical parameters have previously been shown to be the neck-shaft angle (NSA) and the cortical thickness at the femoral shaft or calcar femoris $[9,12]$, which parameters are well represented in our models.

The relative distribution of the cortical and trabecular compartments at the upper femur is subject to change with aging, and it is likely that this distribution affects the susceptibility to different fracture types [34]. The segmentation procedure used here was designed to identify the individual distribution of the two compartments and to include it in the FE model.

Previous studies $[26,35,36]$ used DXA to generate 2D FE models of the proximal femur, taking into account also the BMD of the bone. However, DXA cannot be used to accurately identify the cortical thickness of the femoral shaft or calcar femoris, geometric factors that have been identified as being important in predicting the fracture type. Use of femora in vitro allowed us to compare our FE models with experimental fractures, showing good predictive ability for the fracture type even without BMD, if geometric information obtainable from radiographs with appropriate resolution is used.

There is only limited previous information on the ability of FE models to predict hip fracture type during a fall. Keyak et al. [18] used a 3D model to predict experimental fracture location, with fracture type prediction accuracy of $79 \%$ (11 out of 14). Thus, the present quite simple 2D model appears to give quite similar fracture type prediction in comparison with the 3D model, even if there was some difference in the testing setup. Our setup produced more cervical fractures, which might explain our somewhat higher prediction accuracy. However, more studies are needed for relevant comparison of the accuracy of 2D and 3D models.

Using models with different material properties for the different regions of trabecular bone improved the accuracy of the method. This suggests that, apart from the geometry of the bone and the cortex, the trabecular density distribution has some impact on the fracture type. We can only speculate why in some cases a cervical fracture was predicted but a trochanteric one occurred, and vice versa. In some bones, the prediction of the hip fracture type was not straight forward, due to ambiguous stress patterns. Thus, for a femur with an individual anatomy with quite equal susceptibility for cervical and trochanteric fractures, the trabecular fine structure will define the final fracture type. Additionally, it is possible that here the minimum cortical thickness of $1 \mathrm{~mm}$ in the model overestimates the impact of cortical bone on the strength of bone in the neck area.

It has been shown earlier [37] that soft tissue might have a role in the decrease of hip fracture risk during a fall by attenuating the impact force. Our models applied a constant thickness for the soft tissue as in our experimental protocol, but variation between patients concerning this thickness might also affect the resulting fracture type during a fall.

Even if the prediction accuracy of fracture type obtained from our 2D models was relatively good, some limitations remain. (1) The use of 2D models was not able to reflect the geometrical variation along the anteroposterior axis. (2) Our models did not take into account the distribution or fine structure of trabecular bone. The Young's modulus values used in the models were taken from literature and were not representative of the subject-specific density and material properties. This should be improved in future studies by personalization of the material property distribution. (3) The rotational angle during loading had to be ignored as well due to the limitation of 2D data. It has been shown in a previous study [38] that a slight variation in loading angle can affect the fracture load due to differences in structural capacity of the femur depending on the fall orientation. However, all the radiographic pictures had been taken using equal protocol with a slight internal rotation.

In conclusion, this study provides an efficient, less timeconsuming tool for fracture type prediction applicable at lower cost and with a smaller radiation dose than required for CT. Our results suggest that the cervical and trochanteric hip fractures can be discriminated using a relatively simple 2D model based on standard radiography. Based on the current experimental findings, the predictive power of these models should now be tested in clinical studies.

\section{ACKNOWLEDGEMENTS}

This study was financially supported by the Finnish Funding Agency for Technology and Innovation, the International Graduate School in Biomedical Engineering and Medical Physics, the Finnish Cultural Foundation, North Ostrobothnia Regional Fund, and Deutsche Forschungsgemeinschaft Grant DFG LO 730/2-2. 


\section{REFERENCES}

[1] Cummings SR, Nevitt MC, Browner WS, et al. Risk factors for hip fracture in white women. Study of Osteoporotic Fractures Research Group. N Engl J Med 1995; 332: 767-73.

[2] Cheng X, Li J, Lu Y, Keyak J, Lang T. Proximal femoral density and geometry measurements by quantitative computed tomography: association with hip fracture. Bone 2007; 40: 169-74.

[3] Johnell O, Kanis JA, Oden A, et al. Mortality after osteoporotic fractures. Osteoporos Int 2004; $15: 38-42$.

[4] Kanis JA. Diagnosis of osteoporosis and assessment of fracture risk. Lancet 2002; 359: 1929-36.

[5] Schuit SC, van der Klift M, Weel AE, et al. Fracture incidence and association with bone mineral density in elderly men and women: the Rotterdam Study. Bone 2004; 34: 195-202.

[6] Ota T, Yamamoto I, Morita R. Fracture simulation of the femoral bone using the finite-element method: how a fracture initiates and proceeds. J Bone Miner Metab 1999; 17: 108-12.

[7] Partanen J, Heikkinen J, Jämsä T, Jalovaara P. Characteristics of lifetime factors, bone metabolism, and bone mineral density in patients with hip fracture. J Bone Miner Metab 2002; 20: 367-75.

[8] Pulkkinen P, Eckstein F, Lochmüller EM, Kuhn V, Jämsä T. Association of geometric factors and failure load level with the distribution of cervical $v s$. trochanteric hip fractures. J Bone Miner Res 2006; 21: 895-901.

[9] Partanen J, Jämsä T, Jalovaara P. Influence of the upper femur and pelvic geometry on the risk and type of hip fractures. J Bone Miner Res 2001; 16: 1540-6.

[10] Gnudi S, Ripamonti C, Lisi L, Fini M, Giardino R, Giavaresi G. Proximal femur geometry to detect and distinguish femoral neck fractures from trochanteric fractures in postmenopausal women. Osteoporos Int 2002; 13: 69-73.

[11] Mautalen CA, Vega EM, Einhorn TA. Are the etiologies of cervical and trochanteric hip fractures different? Bone 1996; 18(3 Suppl): S133-7.

[12] Pulkkinen P, Partanen J, Jalovaara P, Jämsä T. Combination of bone mineral density and upper femur geometry improves the prediction of hip fracture. Osteoporos Int 2004; 15: 274-80.

[13] Keyak JH, Falkinstein Y. Comparison of in situ and in vitro CT scan-based finite element model predictions of proximal femoral fracture load. Med Eng Phys 2003; 25: 781-7.

[14] Keyak JH, Skinner HB, Fleming JA. Effect of force direction on femoral fracture load for two types of loading conditions. J Orthop Res 2001; 19: 539-44

[15] Verhulp E, van Rietbergen B, Huiskes R. Load distribution in the healthy and osteoporotic human proximal femur during a fall to the side. Bone 2008; 42: 30-5.

[16] Cody DD, Gross GJ, Hou FJ, Spencer HJ, Goldstein SA, Fyhrie DP. Femoral strength is better predicted by finite element models than QCT and DXA. J Biomech 1999; 32: 1013-20.

[17] Rudman KE, Aspden RM, Meakin JR. Compression or tension? The stress distribution in the proximal femur. Biomed Eng Online 2006; 20: 5-12.

[18] Keyak JH, Rossi SA, Jones KA, Les CM, Skinner HB. Prediction of fracture location in the proximal femur using finite element models. Med Eng Phys 2001; 23: 657-64.

[19] Keyak JH, Rossi SA. Prediction of femoral fracture load using finite element models: an examination of stress- and strain-based failure theories. J Biomech 2000; 33: 209-14.

[20] Bessho M, Ohnishi I, Okazaki H, et al. Prediction of the strength and fracture location of the femoral neck by CT-based finiteelement method: a preliminary study on patients with hip fracture. J Orthop Sci 2004; 9: 545-50.

[21] Schileo E, Taddei F, Cristofolini L, Viceconti M. Subject-specific finite element models implementing a maximum principal strain criterion are able to estimate failure risk and fracture location on human femurs tested in vitro. J Biomech 2008; 41: 356-67.

[22] Voo L, Armand M, Kleinberger M. Stress Fracture Risk Analysis of the Human Femur Based on Computational Biomechanics. Johns Hopkins apl technical digest 2004; 25: 223-30.

[23] Nikander R, Kannus P, Dastidar P, et al. Targeted exercises against hip fragility. Osteoporos Int, Published online Nov 11, 2008; Available as DOI 10.1007/s00198-008-0785-x.

[24] Keaveny TM, Hoffmann PF, Singh M, et al. Femoral bone strength and its relation to cortical and trabecular changes after treatment with $\mathrm{PTH}$, alendronate, and their combination as assessed by finite element analysis of quantitative CT scans. J Bone Miner Res 2008; 23: $1974-82$.

[25] Brownbill RA, Lindsey C, Crncevic-Orlic Z, Ilich JZ. Dual hip bone mineral density in postmenopausal women: geometry and effect of physical activity. Calcif Tissue Int 2003; 73: 217-24

[26] Testi D, Viceconti M, Cappello A, Gnudi S. Prediction of hip fracture can be significantly improved by a single biomedical indicator. Ann Biomed Eng 2002; 30: 801-7.

[27] Testi D, Cappello A, Sgallari F, Rumpf M, Viceconti M. A new software for prediction of femoral neck fractures. Comput Methods Programs Biomed 2004; 75: 141-5.

[28] Eckstein F, Wunderer C, Boehm H, et al. Reproducibility and side differences of mechanical tests for determining the structural strength of the proximal femur. J Bone Miner Res 2004; 19: 37985 .

[29] Wei HW, Sun SS, Jao SH, Yeh CR, Cheng CK. The influence of mechanical properties of subchondral plate, femoral head and neck on dynamic stress distribution of the articular cartilage. Med Eng Phys 2005; 27: 295-304.

[30] Sievänen H, Kannus P, Nieminen V, Heinonen A, Oja P, Vuori I. Estimation of various mechanical characteristics of human bones using dual energy X-ray absorptiometry: methodology and precision. Bone 1996; 18(1 Suppl): S17-27.

[31] Martens M, Van Audekercke R, Delport P, De Meester P, Mulier JC. The mechanical characteristics of cancellous bone at the upper femoral region. J Biomech 1983; 16: 971-83.

[32] Viceconti M. A comparative study on different methods of automatic mesh generation of human femurs. Med Eng Phys 1998; 20: $1-10$

[33] Lengsfeld M, Schmitt J, Alter P, Kaminsky J, Leppek R Comparison of geometry-based and CT voxel-based finite element modelling and experimental validation. Med Eng Phys 1998; 20: $515-22$.

[34] Meta M, Lu Y, Keyak JH, Lang T. Young-elderly differences in bone density, geometry and strength indices depend on proximal femur sub-region: a cross sectional study in Caucasian-American women. Bone 2006; 39: 152-8.

[35] Testi D, Viceconti M, Baruffaldi F, Cappello A. Risk of fracture in elderly patients: a new predictive index based on bone mineral density and finite element analysis. Comput Methods Programs Biomed 1999; 60: 23-33.

[36] Yang L, Peel N, Clowes JA, McCloskey EV, Eastell R. Use of DXA-based structural engineering models of the proximal femur to discriminate hip fracture. J Bone Miner Res 2009; 24: 33-42.

[37] Bouxsein ML, Szulc P, Munoz F, Thrall E, Sornay-Rendu E, Delmas PD. Contribution of trochanteric soft tissues to fall force estimates, the factor of risk, and prediction of hip fracture risk. J Bone Miner Res 2007; 22: 825-31.

[38] Pinilla TP, Boardman KC, Bouxsein ML, Myers ER, Hayes WC. Impact direction from a fall influences the failure load of the proximal femur as much as age-related bone loss. Calcif Tissue Int 1996; 58: 231-5

Received: February 27, 2009

Revised: May 13, 2009

Accepted: May 28, 2009

(C) Thevenot et al.; Licensee Bentham Open.

This is an open access article licensed under the terms of the Creative Commons Attribution Non-Commercial License (http://creativecommons.org/licenses/by$\mathrm{nc} / 3.0 /$ ), which permits unrestricted, non-commercial use, distribution and reproduction in any medium, provided the work is properly cited. 